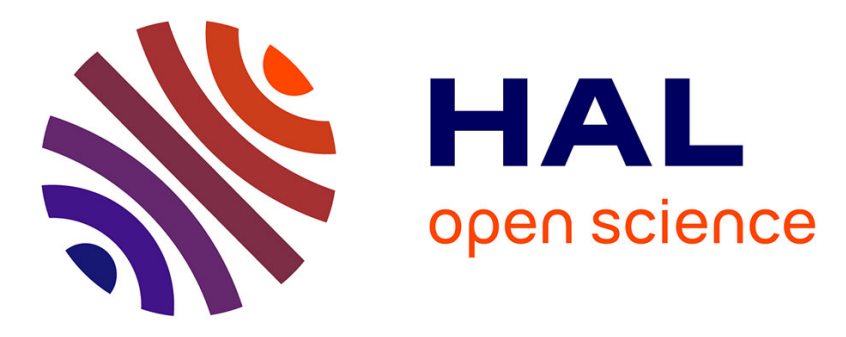

\title{
Temperature Unmasks Allosteric Propensity in a Thermophilic Malate Dehydrogenase via Dewetting and Collapse
}

\author{
Marina Katava, Massimo Marchi, Dominique D Madern, Michael Sztucki, \\ Marco Maccarini, Fabio Sterpone
}

\section{To cite this version:}

Marina Katava, Massimo Marchi, Dominique D Madern, Michael Sztucki, Marco Maccarini, et al.. Temperature Unmasks Allosteric Propensity in a Thermophilic Malate Dehydrogenase via Dewetting and Collapse. Journal of Physical Chemistry B, 2020, 124 (6), pp.1001-1008. 10.1021/acs.jpcb.9b10776 . hal-03008190

\section{HAL Id: hal-03008190 https://hal.science/hal-03008190}

Submitted on 16 Nov 2020

HAL is a multi-disciplinary open access archive for the deposit and dissemination of scientific research documents, whether they are published or not. The documents may come from teaching and research institutions in France or abroad, or from public or private research centers.
L'archive ouverte pluridisciplinaire HAL, est destinée au dépôt et à la diffusion de documents scientifiques de niveau recherche, publiés ou non, émanant des établissements d'enseignement et de recherche français ou étrangers, des laboratoires publics ou privés. 


\section{Temperature Unmasks Allosteric Propensity in a}

\section{Thermophilic Malate Dehydrogenase via Dewetting and Collapse}

M. Katava, ${ }^{\dagger}$ M. Marchi, ${ }^{\complement}$ D. Madern,,${ }^{*}$ M. Sztucki,,$\|$ M. Maccarini, ${ }^{\perp}$ and F. Sterpone, ${ }^{* \dagger}$

$\dagger$ CNRS, Univ. de Paris, UPR9080, Laboratoire de Biochimie Théorique, 13 rue Pierre et Marie Curie, F-75005, Paris, France

$\ddagger$ Institut de Biologie Physico-Chimique-Fondation Edmond de Rothschild, PSL Research University, Paris, France

ICommissariat à l'Energie Atomique DRF/Joliot/SB2SM Centre d'Etudes de Saclay 91191 Gif sur Yvette Cedex

$\S$ Univ. Grenoble Alpes, CEA. CNRS, IBS, 38000 Grenoble, France

||ESRF - The European Synchrotron Radiation Facility, 71 Avenue des Martyrs, 38000 Grenoble, France

$\perp$ Univ. Grenoble Alpes, Laboratoire TIMC/IMAG UMR CNRS 5525 Grenoble, France

E-mail: dominique.madern@ibs.fr; fabio.sterpone@ibpc.fr 


\begin{abstract}
In this work we combine experiments and molecular simulations to unveil the hidden allosteric propensity of a thermophilic malate dehydrogenase protein (MDH). We provide evidence that at its working temperature the non-allosteric $\mathrm{MDH}$ takes a compact structure because of internal dewetting, and reorganizes the active state toward functional conformations similarly to its homologous allosteric LDHs. Moreover, a single-point mutation confers the MDH a cooperative behaviour that mimics an allosteric LDH. Our work not only demonstrates that thermophilic MDHs use temperature as an external parameter to regulate its functionality in a similar way allosteric LDHs use substrates/cofactors binding, but also shows that the scaffold of MDHs possesses an intrinsic and hidden allosteric potentiality.
\end{abstract}

\title{
Introduction
}

The functionality of many proteins is regulated by allostery, a mechanism where the binding affinity or the catalytic efficiency of the enzyme are changed via long-range communication by the binding of an effector molecule in a region located away from the active site ${ }^{1-3}$. For instance, in multi-domain proteins, the allosteric regulation occurs as a cooperative effect: the binding of the substrate in one domain affects the functional capability of the active sites located in the other domains. Therefore, understanding how long range communication occurs in allosteric proteins can reveal the intimate relationship between local and global motions in proteins.

It is not yet clear how allostery emerged as a functional regulator mechanism during evolution ${ }^{4}$. To gain insight in this aspect of allostery, it is strategic to compare homologous proteins sharing a similar structural scaffold but different regulative mechanisms. Because of the great variety of homologues, NAD(P)H-dependent Malate dehydrogenases (MDHs) (EC 1.1.1.37) and NADH-dependent Lactate dehydrogenases (LDHs) (EC 1.1.1.27) are paradigm to investigate 
the molecular mechanisms underlying adaptation and evolution of enzymes ${ }^{5-8}$. MDHs and LDHs are closely related enzymes which share a common fold and catalyze the interconversion of 2-ketoacids to the corresponding 2-hydroxyacids by using a similar mechanism ${ }^{9-12}$. MDHs operate in the citric acid cycle by converting oxaloacetate (OAA) into malate. LDHs play an important role in the last step of glycolysis where lactate is produced from pyruvate (Pyr). In the $\mathrm{MDH} / \mathrm{LDH}$ superfamily, the rate-limiting step during catalysis is associated to the conformational change of the mobile loop that covers the catalytic site and proceeds from an open to a closed $\operatorname{state}^{13}$ (see SI Fig. S1). Mutations on this loop have revealed the identity and location of the substrate-discriminating residue, which is an arginine or a glutamine in MDHs and LDHs, respectively ${ }^{14-16}$. However, other residues are important for the specificity of the MDH vs LDH activity (see SI).

In this work, we focus on two groups of the super-family, the tetrameric LDHs and their closely related MDHs (LDH-like MDH) ${ }^{8,17,18}$. In LDHs, most of the bacterial enzymes exhibit homotropic (by pyruvate) and heterotropic (via fructose 1,6-biphosphate, FBP) activation. The associated allosteric cooperativity is sustained by a global conformational change that triggers the inter-domain communication. In fact, structurally the holo state manifests a compact quaternary structure, while the apo protein is more expanded ${ }^{7,19}$. On the contrary, eukaryotic LDHs and MDHs are considered non-allosteric. The divergence from a common ancestral gene, and the presence of allosteric and non-allosteric enzymes in the super-family make LDH-like MDHs and LDHs a perfect model to investigate the evolutionary emergence of allostery as a mechanism of functional regulation ${ }^{20-22}$. In a previous work based on the combination of neutron scattering experiments and molecular simulations, it was shown that the non-allosteric LDH from rabbit uses temperature to reorganize its catalytic site in a similar manner as allosteric LDHs do upon co-factor binding, and changing from the apo to holo state ${ }^{23}$. More recently, a biochemical and structural study using a LDH-like MDH from a hyperthermophilic archaea has revealed that this enzyme, which was considered as non-allosteric, may exhibit a 
homotropic activation capacity in certain conditions ${ }^{21}$.

Inspired by these studies, we combine results from small angle X-ray scattering (SAXS), molecular dynamics simulations, and biochemistry to pursue the search of hidden allosteric capability in the LDH-like group of MDHs. Namely, we have studied the thermal response of the MDH enzyme from the hyperthermophilic archaean Methanocaldococcus jannaschii ( $M$. jan) in order to unveil how temperature acts on the protein functional conformations ${ }^{24}{ }^{2} M$. jan $\mathrm{MDH}$ is an ideal study-case since the crystal structure of its apo state (PDB code $1 \mathrm{HYG}$ ) shows an expanded conformation similar to that resolved for the allosteric apo LDHs, see SI Fig. S1.

Our results clearly show that for this thermophilic enzyme temperature acts as a regulatory factor allowing the protein to access functional conformations only in the optimal high temperature regime. Remarkably, this temperature-dependent conformational shift is not only a change in flexibility that is often invoked to explain enzyme adaptation to high temperature ${ }^{25,26}$, but is also associated to a counterintuitive collapse of the quaternary structure. The latter is driven by the joint action of internal water escape and local hydrophobic and ionic interactions at high temperature. The collapse is accompanied by local restructuring of the catalytic site, and recalls the contraction observed in allosteric LDHs upon effector binding. In addition, we use a a single-point mutation on the mobile active site loop to show that the mutated $M$. jan $\mathrm{MDH}$ is not only capable of recognising the pyruvate as substrate, but that it also manifests homotropic allostery. Altogether our findings support the idea that the tetrameric scaffold of LDH-like MDHs/LDHs has been evolutionary tailored for specific function and regulation, and additionally reveal a hidden allosteric propensity in some LDH-like MDHs. 


\section{Methods}

\section{Enzymatic assay and protein determination}

The activity of $M$. jan $\mathrm{MDH}$ for reduction of oxaloacetate to malate was carried out at $343 \mathrm{~K}$ in $500 \mu \mathrm{L}$ of $50 \mathrm{mM}$ phosphate buffer pH 7.0 containing $50 \mathrm{mM} \mathrm{NaCl}$. Assays with the mutant R86Q using pyruvate as substrate were done in $500 \mu \mathrm{L}$ of 2 -(N-morpholino) ethane sulfonic acid (MES) pH 6.0 and supplemented with $50 \mathrm{mM} \mathrm{NaCl}$. The reaction was monitored at $340 \mathrm{~nm}$ by following the oxidation of NADPH $(0.5 \mathrm{mM})$ on a Jasco 540 spectrophotometer. To record the enzymatic profile of $M$. jan $\mathrm{MDH}$ and mutant, various substrates concentrations were tested. The measurements were done in triplicates. The data were analysed using Michaelis-Menten or allosteric sigmoidal equations in GraphPad Prism version 7.03. The protein concentration was estimated from the absorbance at $280 \mathrm{~nm}$ using a nanodrop thermofischer. The server https://web.expasy.org/protparam/ (Gasteiger et al., 2005) was used to compute a molecular weight of $34.6 \mathrm{kDa}$ and a theoretical extinction coefficient of $9190 \mathrm{M}^{-1} \mathrm{~cm}^{-1}$.

\section{Cloning and activity of $M$. jan MDH and mutant.}

A gene encoding the wild-type (Wt) malate dehydrogenase of $M$. jan and the mutant R86Q (linear numbering) with codons optimised for expression in E. coli were synthesised and sub cloned into pET-20a by Gencust. Compared to the wild type MDH, the properties of R86Q mutant show that it is a less efficient enzyme because of i) a decrease of specific activity and ii) an increase of the substrate affinity parameter value indicating a decrease of affinity. The specific activity of $\mathrm{WT} \mathrm{MDH}$ is $888 \mathrm{U} / \mathrm{mg}$ while that of R86Q mutant is $15 \mathrm{U} / \mathrm{mg}$. The substrate affinity $K_{m}$ of WT MDH is $0.3 \mathrm{mM}$ with oxaloacetate and $S_{0.5}$ of R86Q mutant is 19 mM with pyruvate. 


\section{Protein expression and purification.}

E. coli BL21 DE competent cells transformed with pET-20 expression vector encoding the Wt and the mutant MDH genes were selected by growth on LB agar plates containing $100 \mathrm{\mu g} \mathrm{mL} \mathrm{m}^{-1}$ ampicillin. A single colony was grown overnight at $37^{\circ} \mathrm{C}$ in $50 \mathrm{~mL} \mathrm{LB}$ medium at the same concentration of antibiotic. $20 \mathrm{~mL}$ of these cultures were then used for inoculation of a 2 liter LB medium containing $100 \mu \mathrm{g} \mathrm{mL} \mathrm{m}^{-1}$ ampicillin. The cells were cultivated at $37^{\circ} \mathrm{C}$ until an OD600 of 0.6 was reached. Isopropyl B-D-1-thiogalactopyranoside (IPTG) was added at a final concentration of $0.5 \mathrm{mM}$ to induce expression and the culture was incubated for 4 hours at $37^{\circ} \mathrm{C}$. Bacterial cells were harvested by centrifugation at $6000 \mathrm{~g}$ for 20 minutes at $4^{\circ} \mathrm{C}$. The pellets were suspended in $40 \mathrm{~mL}$ of $50 \mathrm{mM}$ Tris- $\mathrm{HCl} \mathrm{pH} 7.4$ containing $50 \mathrm{mM} \mathrm{NaCl}$ (Buffer A). Prior cell disruption, $5 \mathrm{\mu g} \mathrm{mL} \mathrm{mL}^{-1}$ of DNAse and $\mathrm{MgCl}_{2}$ to final concentration of $15 \mathrm{mM}$ was added to the cell suspension. The preparations were cooled at $4{ }^{\circ} \mathrm{C}$ and lysed by sonication (Branson sonicator). Six cycles of continuous sonication at $50 \%$ amplitude were applied during $30 \mathrm{~s}$. Between each pulse, the solutions were kept on ice for one minute. The extract was then centrifuged at $13000 \mathrm{~g}$ for $30 \mathrm{~min}$ at $4{ }^{\circ} \mathrm{C}$. The supernatant was further incubated at $75^{\circ} \mathrm{C}$ for $30 \mathrm{~min}$ and the thermally unfolded proteins were removed by centrifugation. The extract were loaded on a Q sepharose column $(2 \times 10 \mathrm{~cm})$ equilibrated with Buffer A. Wt M. jan MDH and the mutant were eluted with a linear gradient from 0 to $0.8 \mathrm{M} \mathrm{NaCl}$ in Buffer A. The active fractions were pooled, concentrated and loaded on a Superpose 12 gel-filtration column (GE Healthcare) and eluted with Buffer A. The purity of the enzymes was checked by SDS gel electrophoresis.

\section{Sample preparation for SAXS experiments.}

The various Wt $M$. jan $\mathrm{MDH}$ samples were dialyzed 10 times against Tris- $\mathrm{HCl}$ buffered at $\mathrm{pH} 7$ in $50 \mathrm{mM} \mathrm{NaCl}$ in pure $\mathrm{D}_{2} \mathrm{O}$ to reach a $\%$ of $\mathrm{D}_{2} \mathrm{O}$ higher than $98 \%$. Appropriate $\mathrm{pH}$ adjustments in $\mathrm{D}_{2} \mathrm{O}$ were done accordingly to $\mathrm{pD}=\mathrm{pH}+0.4$. The protein was concentrated 
up to $100 \mathrm{mg} \mathrm{mL}^{-1}$ in this buffer using an Amicon ultrafiltration PM30 system prior SAXS experiments. The last flow-through solution was used to monitor the scattering of the buffer. While the preparation of the protein in $\mathrm{D}_{2} \mathrm{O}$ does not affect the SAXS experiments reported in this work, the same samples were used to perform neutron scattering experiments that will be reported in a separate work.

\section{Small-Angle X-ray Scattering}

SAXS experiments were performed at the TRUSAXS beamline ID02 at ESRF (Grenoble, France $)^{27}$. Two-dimensional scattering patterns were collected using a Rayonix MX-170HS CCD detector operated in 6x6 binning in order to reduce readout noise. The X-ray energy was set to $12460 \mathrm{eV}$ and the detector was placed $2.5 \mathrm{~m}$ from the sample in an evacuated flight tube. The recorded scattering patterns have been corrected for detector artifacts, normalized to absolute intensity and azimuthally averaged to obtain the intensity profile I(q) [with scattering wave vector $Q=(4 \pi / \lambda) \sin (\vartheta / 2), \lambda \approx 1 \AA$ being the wavelength and $\vartheta$ the scattering angle]. Samples were loaded in a Peltier thermostated flow-through capillary sample holder (2mm diameter) to allow accurate background subtraction.

\section{Molecular Dynamics}

MD simulations of $M$. jan $\mathrm{MDH}$ (pdb code $1 \mathrm{HYG}$ ) were carried out using different force fields (ff), charmm27 (c27) ${ }^{28}$, charmm36 (c36) ${ }^{29}$, and amber03 ${ }^{30}$ and at different temperatures. We first performed long MD simulations using the c27 ff in the NPT ensemble at ambient pressure and in a range of temperatures between $283 \mathrm{~K}$ and $360 \mathrm{~K}$ (for this simulation we used the NAMD code ${ }^{31}$ ). The system was initially placed in a cubic simulation box of size $L=110 \AA$. At each temperature the simulation was extended for about 500-600 ns. For this system and at the lowest temperature, we performed extra simulations using the c36 and the amber03 ffs (for these simulations we used the Gromacs code ${ }^{32}$ ). In order to inspect the size effect, we also 
considered a large cubic box with average size $L=127-130 \AA$. This latter set-up based on the $\mathrm{c} 36 \mathrm{ff}$ was used to investigate in detail the molecular properties of the temperature induced collapse. In the large box, the system contains 62002 water molecules and 16 ions, for a total system size of 205,866 particles. For this system, the simulations were carried out at $283 \mathrm{~K}$, $343 \mathrm{~K}$, and $360 \mathrm{~K}$, and extended for about 300-400 ns each.

\section{Results}

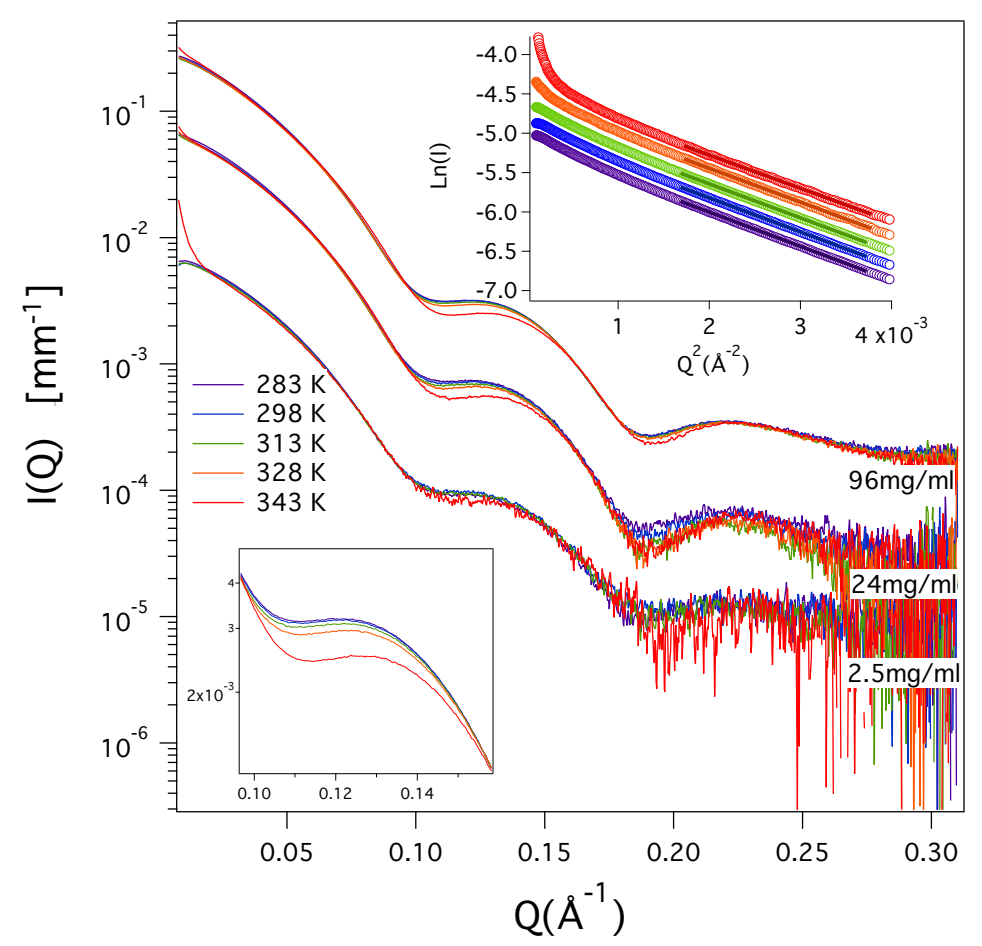

Figure 1: SAXS scattering curves as a function of $Q$ measured at three different protein concentrations and at five temperatures. In the top inset graph we report the Guinier plot for the lower concentration, $c=25 \mathrm{mg} / \mathrm{mL}$. In the bottom inset graph, for the highest concentration, we zoom the SAXS curves in order to magnify the temperature effect on the shoulder of the curve. 


\section{Small Angle X-Ray Scattering}

We first present results from SAXS experiments, which reveal some of the structural properties of the system. Figure 1 depicts the SAXS scattering curve measured for solutions of $M$. jan $\mathrm{MDH}$ as a function of $Q$ at different concentrations and temperatures. Although the intensity of the curves increases at higher concentration due to the presence of more scatterers, the SAXS profiles always show the same main features: two structural shoulders in the range $0.1-0.15 \AA^{-1}$ and $0.2-0.25 \AA^{-1}$. These structural features correspond to length-scales of $42-62 \AA$ (the size of the quaternary structure of the protein), and 25-31 $\AA$ (the size of the each of the four subunits), respectively. A close inspection of the SAXS curves reveals a remarkable change in the first shoulder at a temperature of $343 \mathrm{~K}$, this change being more evident at higher concentrations in view of the better signal. At this temperature, the shoulder shifts toward higher $Q$-values, suggesting a rearrangement of the relative position of the subunits in the protein into a more compact form.

In the top inset of Figure 1 the logarithm of the SAXS intensity is reported as a function of $Q^{2}$ for the lowest concentration. At low values of $Q^{2}$, the measurements at high temperature are affected by partial aggregation of the proteins in the solution that alters the linearity expected at this $Q$-scale. However, at a higher $Q^{2}$ range between 1.7 and $3.7 \cdot 10^{-3} \AA^{-2}$, the trend in the $\ln I$ vs $Q^{2}$ shows a perfect linear trend. Although we are outside the strict range of validity of the Guinier regime $\left(R_{g} Q<1.3\right)^{33}$, the angular coefficient of this linear scaling can still be associated to a characteristic length-scale of the protein. The temperature induces a change of this characteristic length scale from the value of $36.17 \pm 0.02 \AA$ at $\mathrm{T}=283 \mathrm{~K}$ to $35.58 \pm 0.04 \AA$ at $\mathrm{T}=343 \mathrm{~K}$. This quantitative evidence supports the idea that the protein undergoes a transition toward a more compact form as a result of the temperature increase. 

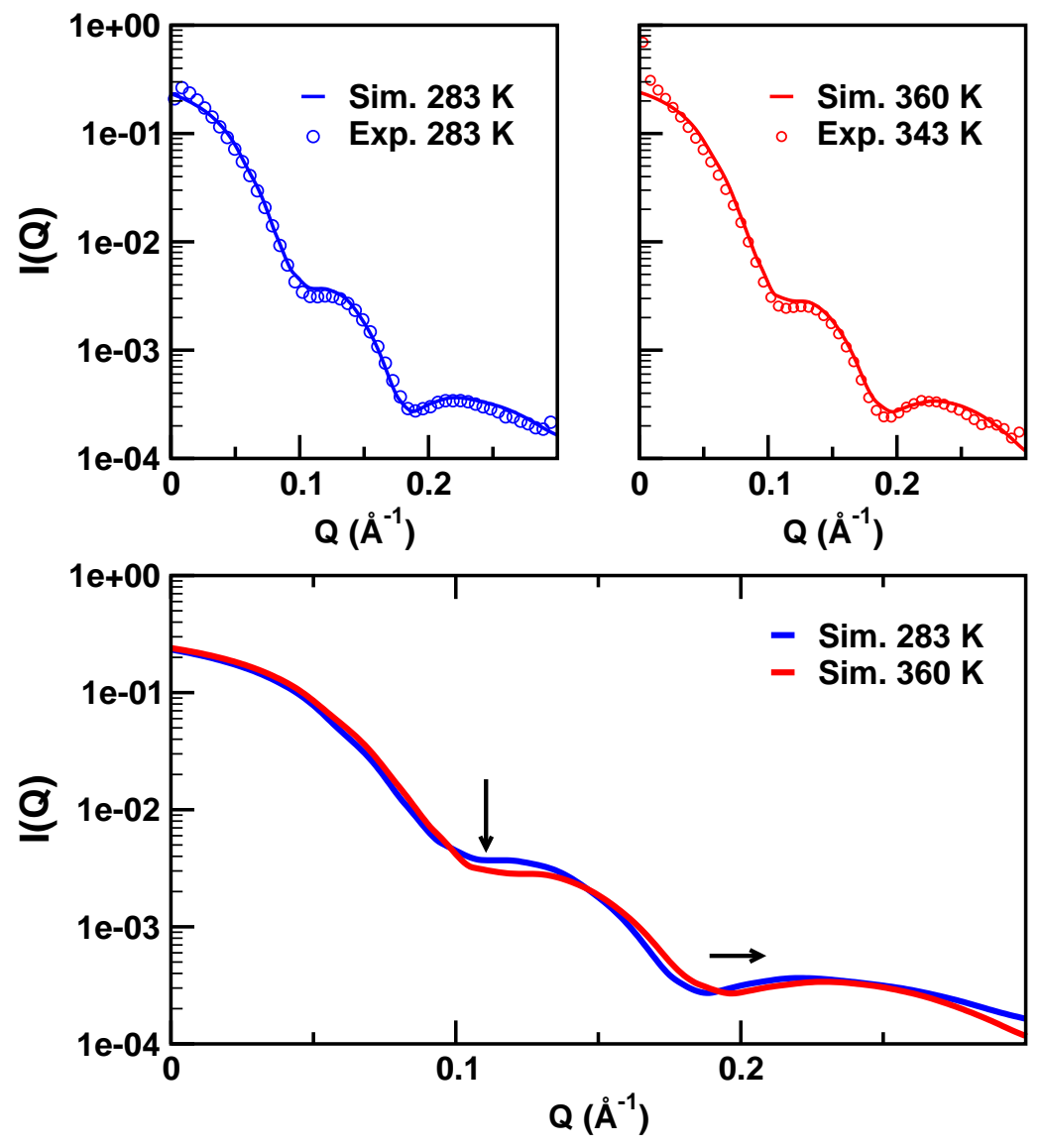

Figure 2: Top panel: Comparison of the SAXS scattering curves obtained via MD simulations and the experimental ones. For sake of clarity, we represented the experimental data using a reduced number of points (skipping factor 1:25). Bottom panel: SAXS spectra derived from MD simulations at different temperatures. The arrows indicate the changes in the spectra due to temperature increase.

\section{Microscopic Insight on Quaternary Structure Collapse}

To dissect the counter-intuitive compaction behaviour at the molecular level, we have performed a set of MD simulations of the MDH protein at different temperatures, and using several force fields and simulation set ups (see the section Methods and SI). To obtain the SAXS profile from MD trajectories we have used the new version of trjSaxs $^{34}$ (the code is available at https://github.com/octupole/openTRJ), which computes the small-angle X-ray scattering $(\mathrm{SAXS})$ intensity of solvated globular and membrane proteins from 3D fast Fourier 
transforms (FFT). The code combines a suitable particle meshing interpolation, similar to the one used in smooth-particle-mesh Ewald for electrostatics ${ }^{35}$, with a uniform solvent density FFT padding scheme to obtain a convenient SAXS spectral resolution. Thus, from the MD runs we reconstructed the SAXS curves that reproduce very precisely the structural features of the experimental spectra, see Figure 2, top panels, and SI Fig. S2. Our tests show that at ambient condition charmm36 (c36) ${ }^{29}$ and amber03 ${ }^{30}$ force fields both provide an excellent agreement with the experimental spectrum, and that the associated curves are identical up to $Q=0.3 \AA^{-1}$, see SI Fig. S2. We also noticed that the effects of the highest experimental temperature $(\mathrm{T}=343 \mathrm{~K})$ - decreased intensity in the region $Q=0.1-0.13 \AA^{-1}$ and shift of the shoulder at $Q \sim 0.22 \AA^{-1}$ - are better reproduced in silico at a higher $\mathrm{T}$ value $(360 \mathrm{~K})$, see Figure 2.

Directly from the sampled conformations of the protein, we have additionally computed the radius of gyration and probed the temperature-induced collapse of the tetramer, see Figure 3.A. At ambient temperature and for all the tested force fields, we find a protein $R_{g} \simeq 33-34 \AA$, this value being about $1-2 \AA$ larger than that associated with its X-ray, probably as effect of the packing in the crystal. When temperature increases, the size of the protein reduces as an effect of the collapse of the quaternary structure. The $R_{g}$ shift, $\Delta R_{g}$, between $283 \mathrm{~K}$ and $343 \mathrm{~K}$ is 0.8-0.5 $\AA$, in strong agreement with the SAXS-derived values. At $360 \mathrm{~K}$, the shift is larger, about $1.3 \AA$ for both charmm27 (c27) ${ }^{28}$ and c36 force fields and does not depend on the size of the simulation cell. While the $R_{g}$ collapse is well reproduced in silico at $\mathrm{T}=343 \mathrm{~K}$, we find that a complete reorganisation of the quaternary structure, as featured by the SAXS spectrum, is only recovered in silico for our simulation time (hundreds of nanoseconds) at $\mathrm{T}=360 \mathrm{~K}$.

The compact state of the quaternary organisation of the tetramer at high temperature is reminiscent of the conformational changes detected in the allosteric LDHs when passing from the apo to the functional holo state, see data in SI Table S1. Moreover, the mesophilic nonallosteric LDH from rabbit that already shows a compact organisation in its crystallographic 

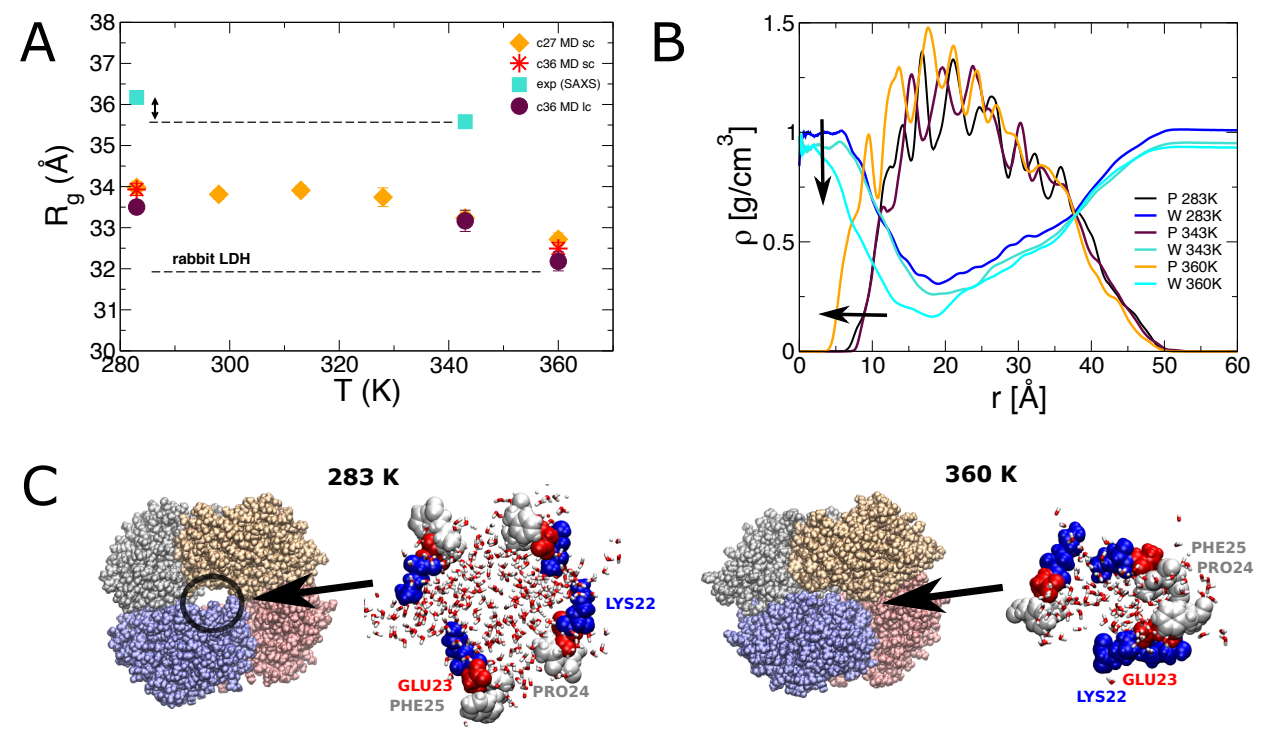

Figure 3: Panel A. Gyration radius $\left(R_{g}\right)$ of the the MDH protein as a function of temperature as extracted from SAXS data and MD simulations. For sake of comparison we also report the value calculated from MD simulation of the non-allosteric LDH protein from rabbit. Panel B. Water and protein densities as a function of the distance from the center of the MDH cavity (simulations based on the c36 force field). Similar results are obtained for simulations based on c27, see SI Fig. S3. Panel C. Molecular view of the quaternary structure of the MDH (chain $\mathrm{A}$ in grey, chain $\mathrm{B}$ in orange, chain $\mathrm{C}$ in blue, and chain $\mathrm{D}$ in red) at $\mathrm{T}=283 \mathrm{~K}$ and $\mathrm{T}=360 \mathrm{~K}$ representing the temperature-induced compact state and the reduction of the internal cavity. The key residues in the internal cavity that trigger the collapse are highlighted. 
structure, when simulated at ambient condition it is associated to a gyration radius of $R_{g} \simeq 32$ $\AA$ (see Ref. ${ }^{23}$ for details); very close to the final value sampled by the thermophilic MDH at its functional high temperature.

Structurally, the domain-packing in the quaternary structure creates an internal cavity that is filled by water. We verified that when the temperature is increased to $360 \mathrm{~K}$ the density of the internal water pool decreases in a much more significant way than the thermal expansion of bulk water. This is depicted in Figure 3.B, where the water and protein density profiles from the center of the cavity are reported. Hence, we can conclude that water escape from the internal cavity triggers the collapse of the domains.

The reduction in volume of the internal cavity is mainly caused by the reorganisation of a cluster of residues (Lys22, Glu23, Pro24, and Phe25) in each chain, see Figure 3.C. In particular, the hydrophobic side chains of Pro24 and Phe25 in adjacent domains get in direct contact, forming a local inter-domain clamp in the internal cavity space, see SI Fig. S4. This is also accompanied by local ion-pairing between the charged residues (Lys22 and Glu23) across the domains. As an effect of the collapse, the number of inter-domain contacts increases by about 20-40\%, see Fig. S5. As shown in the past, inter-domain dewetting and hydrophobic collapse are a driving force in multi-domain protein assembly ${ }^{36}$. In this context, it was recently found that the key residues involved in the allosteric conformational changes in LDHs were also involved in the folding collapse of the same protein ${ }^{37}$.

The protein conformational shifts induced by temperature affect also the fine organisation of the active site. In fact, at high temperature, the loop that acts as gateway for the active site starts exploring closed conformations. This is a necessary condition to bring the substratediscriminating residue Arg86 (102 in the aligned sequence to LDH, see SI Fig. S1) in contact with one of the carboxylate group of the oxaloacetate and trigger the catalysis, see top part of Figure 4. At the same time, we also observe that the side chain of the substrate binding residue Arg154 (171 in the aligned sequence to LDH, see SI Fig. S1), starts exploring an extended 
configuration and points toward the center of the site where it would interact with second carboxylate group of the substrate ${ }^{7,19}$, see bottom part of Figure 4.

\section{Triggering homotropic activation in a $\mathrm{MDH}$}

Having shown that the temperature-dependent functional conformations of $M$. jan MDH share similarities with those regulated by allostery in LDHs, we attempted a final test to verify whether or not the LDH mode of functioning can be implemented in the $M$. jan MDH scaffold. An in vitro single-point mutation was carried out by replacing the catalytic arginine Arg86 (102 in the aligned sequence to $\mathrm{LDH}$ ), located at the gateway loop and responsible of the substrate recognition, into a glutamine (Gln, Q) which is the amino acid always present in the LDHs (SI Fig. S1). The Figure 5.B shows that the MDH R86Q not only recognises and transforms the pyruvate as LDHs, but additionally shows the typical sigmoid form of the homotropic allosteric behaviour, albeit with lower catalytic efficiency (see Methods). The latter was observed by measuring the activity of the enzyme as a function of the substrate concentration. For the sake of comparison, we report in Figure 5.A the activity of the WT as a function of oxaloacetate concentration at the working condition $\sim 343 \mathrm{~K}$.

\section{Conclusion}

The allosteric behaviour of $\mathrm{LDHs}^{38}$ is described by the Monod-Wyman-Changeaux model ${ }^{39}$. In this model, the T-inactive and R-active states exist in equilibrium prior to the binding of the allosteric activator ${ }^{40}$. The allosteric regulation therefore emerges due to changes in the population of the two states caused by the activator. However, probing the equilibrium shift pre- and post- activator's binding is experimentally challenging. X-ray crystallographic structures of LDHs in apo state are found in an open conformation, while when the protein is bound to substrate, the structure is closed and compact even without the presence of allosteric 
loop open

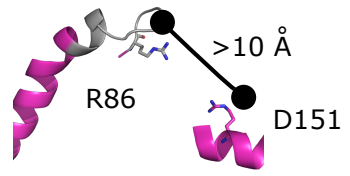

loop closed

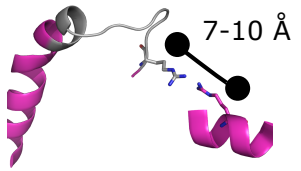

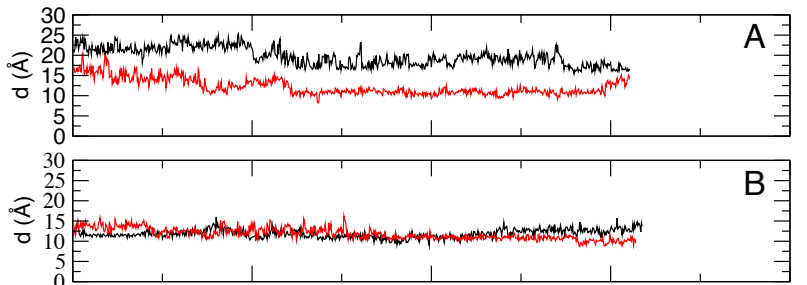
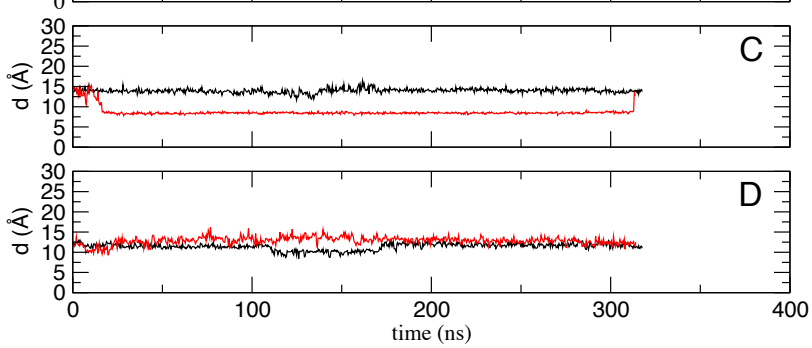

$\mathrm{T}=283 \mathrm{~K} \bigcirc \mathrm{T}=360 \mathrm{~K}$
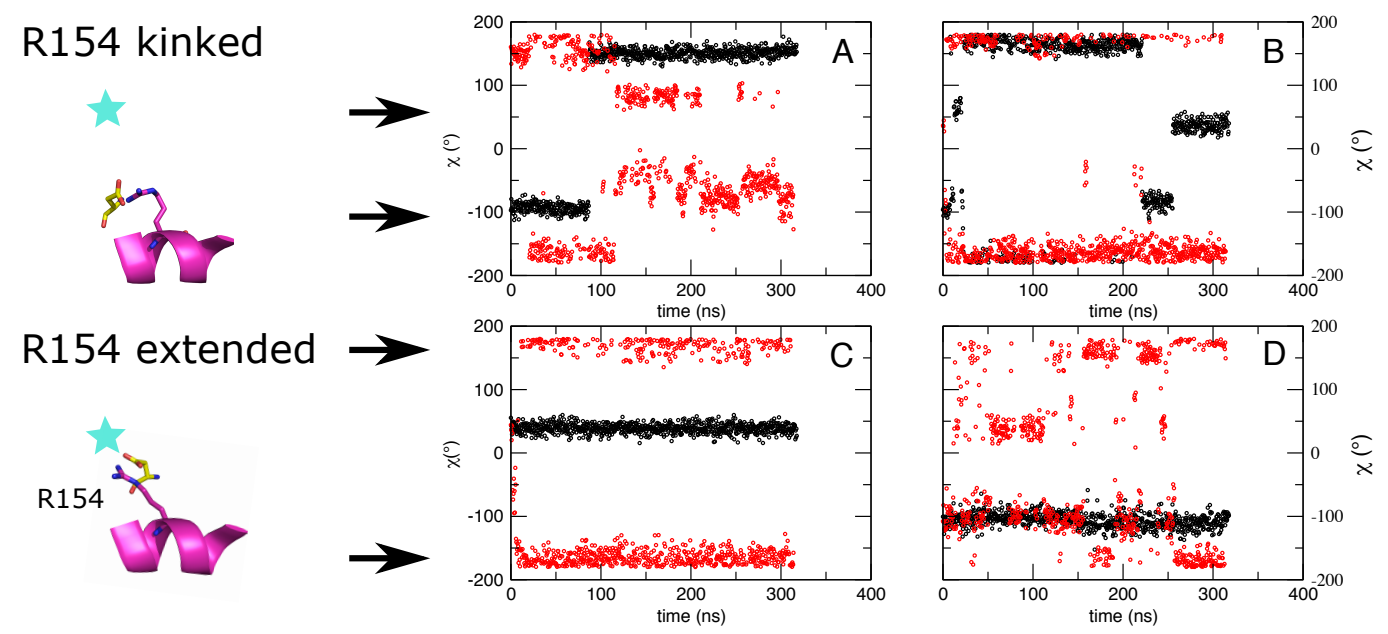

Figure 4: Active site. Reorganisation of the active site caused by the temperature raise from $\mathrm{T}=283 \mathrm{~K}$ to $\mathrm{T}=360 \mathrm{~K}$. In the top part of the figure, the conformation (open/close) of the catalytic loop is monitored considering the distance from the side-chain center-of-mass of two key residues, Arg86 (102 in the aligned sequence to LDH, see Fig. S1) located in the loop and Asp151 located in the catalytic site. The distances are computed for the four A-D domains. In the bottom part of the figure, we monitor the orientation of the catalytic residue Arg154 (171 in the aligned sequence to LDH, see Fig. S1) by considering the side-chain dihedral $\chi$ associated to a kinked or extended configurations. The extended configuration is required in the holo protein to perform catalysis. The configurations are sampled for all the A-D domains. 

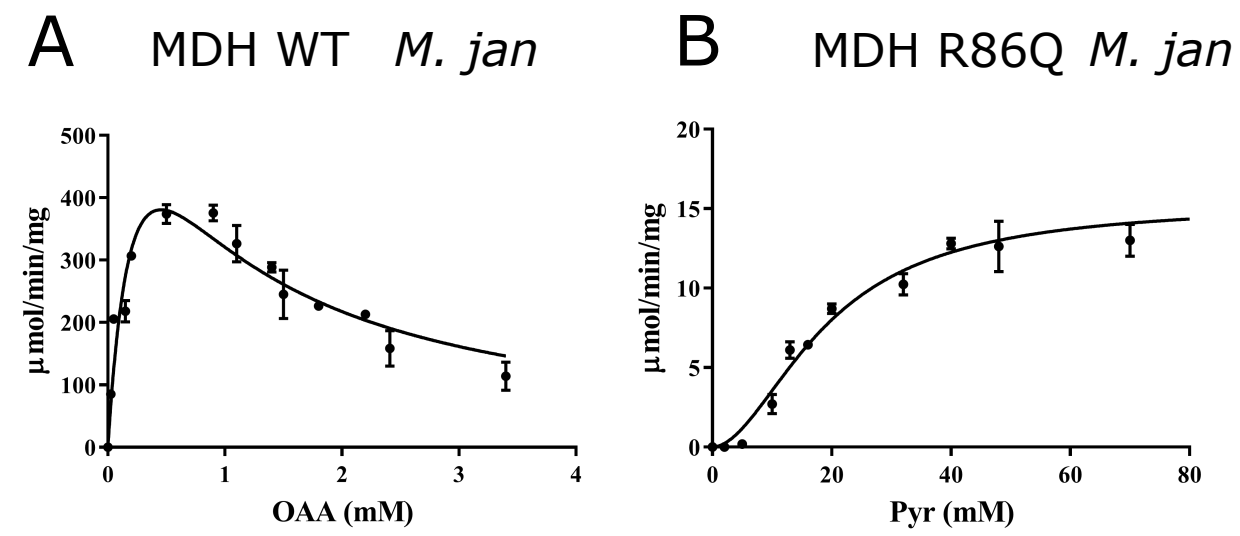

Figure 5: Panel A. Enzymatic activity profiles of $M$. jan MDH wild-type with oxaloacetate. Panel B. Enzymatic activity profiles of R86Q mutant with pyruvate. Reaction velocities were measured in the presence of the indicated concentrations of substrate. Both activities were recorded at $343 \mathrm{~K}$.

effector $\mathrm{FBP}^{7,41}$. The global contraction is shown to be coupled to a reorganisation of the active site $^{42}$.

While structural comparisons show that most of the apo crystal structures of archaeal LDHlike MDHs deposited in the protein data bank strongly resemble the holo (R-active) compact state of LDHs, the apo tetrameric structure of $M$. jan MDH shows an expanded conformation similar to the apo state (T-inactive) of allosteric LDHs, see SI Fig. S1. This unique peculiarity suggests that in order to function, this protein should transform into a compact state similarly to allosteric LDHs. We recall that, to date, MDH $M$. jan structure has only been resolved in the apo state.

Therefore, the SAXS experiments offered a unique opportunity to sample the global structure of $M$. jan $\mathrm{MDH}$ toward its functional high temperature regime where a compact configuration is expected. Combined with MD simulations, we clearly show that the compact state and the functional reorganization of the catalytic site are indeed triggered by temperature increase. M. jan $\mathrm{MDH}$ thus reproduces, under the effect of thermal excitation, the allosteric conformational changes viewed in LDHs. This allosteric propensity of $M$. jan $\mathrm{MDH}$ is further confirmed 
by implementing a single-point mutation capable of altering the substrate recognition from oxaloacetate to pyruvate, and activating allosteric cooperative functional response to substrate concentration. The role of temperature as an alternative regulative external parameter in the LDHs/MDHs evolution has been previously suggested by comparing the binding affinity of the allosteric LDH from T. thermophilus ${ }^{43}$. For this protein, when placed at its high functional temperature $(353 \mathrm{~K})$, the obtained high affinity matches the value recorded at ambient conditions but in presence of the allosteric effector FBP.

This work has clearly identified the internal dewetting and the temperature controlled hydrophobic collapse as the elementary physical-chemical processes underlying the allosteric-like functional conformational changes in M. jan $\mathrm{MDH}$.

\section{Supporting Information Available}

Structural and functional comparison on LDHs/MDHs. SAXS curves from MD using different force fields. Details of MDH structural collapse. This information is available free of charge via the Internet at http://pubs.acs.org.

\section{Acknowledgement}

The research leading to these results has received funding from the ERC (FP7/2007-2013) Grant Agreement no.258748. Part of this work was performed using HPC resources from LBT, GENCI [CINES and TGCC] (Grant x2017/2018/2019/76818). We acknowledge the financial support by ANR (grant "AlloAnc" ANR-16-CE11-0011), the "Initiative d'Excellence" program from the French State (Grant "DYNAMO", ANR-11-LABX-0011-01, and "CACSICE", ANR-11-EQPX0008). We acknowledge the European Synchrotron Radiation Facility (Grenoble,France) for allocation of beam time 


\section{References}

(1) Gunasekaran, K.; Ma, B.; Nussinov, R. Is Allostery an Intrinsic Property of all Dynamic Proteins? Proteins 2004, 5\%, 433-443.

(2) Motlagh, H. N.; Wrabl, J. O.; Li, J.; Hilser, V. The Ensemble Nature of Allostery. Nature 2014, 508, 331-339.

(3) Guo, J.; Zhou, H.-X. Protein Allostery and Conformational Dynamics. Chem. Rev. 2016, $116,6503-6515$.

(4) James, L. C.; Tawfik, D. S. Conformational Diversity and Protein Evolution - a 60-YearOld Hypothesis Revisited. Trends Biochem. Sci. 2003, 28, 361-368.

(5) Fields, P.; Somero, N. G. Hot Spots in Cold Adaptation: Localized Increases in Conformational Flexibility in Lactate Dehydrogenase A4 Orthologs of Antarctic Notothenioid Fishes. Proc. Natl. Acad. Sci. USA 1998, 95, 11476-11481.

(6) Tehei, M.; Madern, D.; Franzetti, B.; Zaccai, G. Neutron Scattering Reveals the Dynamic Basis of Protein Adaptation to Extreme Temperature. J. Biol. Chem. 2005, 280, 4097440979.

(7) Coquelle, N.; Fioravanti, E.; Weik, M.; Vellieux, F.; Madern, D. Activity, Stability and Structural Studies of Lactate Dehydrogenases Adapted to Extreme Thermal Environments. J. Mol. Biol. 2007, 374, 547-562.

(8) Boucher, J.; Jacobowitz, J. R.; Beckett, B. C.; Classen, S.; Theobald, D. L. An AtomicResolution View of Neofunctionalization in the Evolution of Apicomplexan Lactate Dehydrogenases. ELife 2014, 3, e02304.

(9) Holbrook, J.; Liljas, A.; Steindel, S.; Rossmann, M. In The Enzymes, 3rd ed.; Boyer, P. D., Ed.; Academic Press, New York, 1975; Vol. 11; pp 191-292. 
(10) Birktoft, J. J.; Banaszak, L. J. The Presence of a Histidine-Aspartic Acid Pair in the Active Site of 2-Hydroxyacid Dehydrogenases. X-ray Refinement of Cytoplasmic Malate Dehydrogenase. J. Biol. Chem. 1983, 258, 472-482.

(11) A. R. Clarke, D. B. W.; Chia, W. N.; Barstow, D.; Atkinson, T.; Holbrook, J. J. SiteDirected Mutagenesis Reveals Role of Mobile Arginine Residue in Lactate Dehydrogenase Catalysis. Nature 1986, 324, 699-702.

(12) Clarke, A. R.; Wilks, H. M.; Barstow, D. A.; Atkinson, T.; Chia, W. N.; Holbrook, J. J. An Investigation of the Contribution Made by the Carboxylate Group of an Active Site Histidine-Aspartate Couple to Binding and Catalysis in Lactate Dehydrogenase. Biochemistry 1988, 27, 1617-1622.

(13) Waldman, A. D. B.; Hart, K. W.; Clarke, A. R.; Wigley, D. B.; Barstow, D. A.; Atkinson, T.; Chia, W. N.; Holbrook, J. J. The Use of a Genetically Engineered Tryptophan to Identify the Movement of a Domain of B. Stearothermophilus Lactate-Dehydrogenase with the Process which Limits the Steady-State Turnover of the Enzyme. Biochem. Biophys. Res. Com. 1988, 150, 752-759.

(14) Wilks, H. M.; Hart, K. W.; Feeney, R.; Dunn, C. R.; Muirhead, H.; Chia, W. N.; Barstow, D. A.; Atkinson, T.; Clarke, A. R.; Holbrook, J. A Specific, Highly Active Malate Dehydrogenase by Redesign of a Lactate Dehydrogenase Framework. Science 1988, 242, $1541-1544$.

(15) Boernke, W. E.; Millard, C. S.; Stevens, P. W.; Kakar, S. N.; Stevens, F. J.; Donnelly, M. I. Stringency of Substrate Specificity of Escherichia Coli Malate Dehydrogenase. Arch. Biochem. Biophys. 1995, 332, 43-52.

(16) Cendrin, F.; Chroboczek, J.; Zaccai, G.; Eisenberg, H.; Mevarech, M. Cloning, Sequencing and Expression in Escherischia Coli of the Gene Coding for Malate Dehydrogenase of the 
Extremely Halophilic Archaebacterium Haloarcula Marismortui. Biochemistry 1993, 32, $4308-4313$.

(17) Madern, D. Molecular Evolution within the L-Malate and L-Lactate Dehydrogenase SuperFamily. J. Mol. Evol. 2002, 54, 825-40.

(18) D. Madern, M. S. A. G. Z., X. M. Cai Evolution of Cryptosporidium parvum lactate dehydrogenase from malate dehydrogenase by a very recent event of gene duplication. Mol. Biol. Evol. 2004, 21, 489-497.

(19) Iwata, S.; Kamata, K.; Yoshida, S.; Minowa, T.; Ohta, T. T and R States in the Crystals of Bacterial L-Lactate Dehydrogenase Reveal the Mechanism for Allosteric Control. Nat. Struct. Mol. Biol. 1994, 1, 176-185.

(20) Perica, T.; Kondo, Y.; Tiwari, S. P.; McLaughlin, S. H.; Kemplen, K. R.; Zhang, X.; Steward, A.; Reuter, N.; Clarke, J.; Teichmann, S. A. Evolution of Oligomeric State Through Allaosteric Pathways that Mimics Lignad Binding. Science 2014, 346, 1254346.

(21) Roche, J.; Girard, E.; Mas, C.; Madern, D. The Archaeal LDH-Like Malate Dehydrogenase from Ignicoccus Islandicus Displays Dual Substrate Recognition, Hidden Allostery and a non-Canonical Tetrameric Oligomeric Organization. J. Struct. Biol. 2019,

(22) Johnsen, U.; Reinhardt, A.; Landan, G.; Tria, F. D. K.; Turner, J. M.; Davies, C.; Schonheit, P. New Views on an Old Enzyme : Allosteric Regulation and Evolution of Archaeal Pyruvate Kinase. FEBS J. 2019, 286, 2471-2489.

(23) Katava, M.; Maccarini, M.; Villain, G.; Paciaroni, A.; Sztucki, M.; Ivanova, O.; Madern, D.; Sterpone, F. Thermal Activation of 'Allosteric-Like' Large-Scale Motions in a Eukaryotic Lactate Dehydrogenase. Sci. Rep. 2017, 7, 41092. 
(24) Madern, D. The Putative L-Lactate Dehydrogenase from Methanococcus Jannaschii is an NADPH-Dependent L-Malate Dehydrogenase. Mol. Microbiol. 2000, 37, 1515-1520.

(25) Somero, G. N. Proteins and temperature. Ann. Rev. Physiol. 1995, 57, 43-68.

(26) Vieille, C.; Zeikus, G. J. Hyperthermophilic enzymes: Sources, Uses, and Molecular Mechanisms for Thermostability. Microbiol. Mol. Biol. Rev. 2001, 65, 1-43.

(27) Narayanan, T.; Sztucki, M.; Vaerenbergh, P. V.; Leonardon, J.; Gorini, J.; Claustre, L.; Sever, F.; Morse, J.; Boesecke, P. A Multipurpose Instrument for Time-Resolved UltraSmall-Angle and Coherent X-ray Scattering. J. Appl. Cryst. 2018, 51, 1511.

(28) MacKerell, A. D.; Bashford, D.; Bellott,; Dunbrack, R. L.; Evanseck, J. D.; Field, M. J.; Fischer, S.; Gao, J.; Guo, H.; Ha, S. et al. All-Atom Empirical Potential for Molecular Modeling and Dynamics Studies of Proteins. J. Phys. Chem. B 1998, 102, 3586-3616.

(29) Huang, J.; MacKerell, A. D. CHARMM36 All-Atom Additive Protein Force Field: Validation Based on Comparison to NMR Data. J. Comput. Chem. 2013, 34, 2135-2145.

(30) Duan, Y.; Wu, C.; Chowdhury, S.; Lee, M. C.; Xiong, G.; Zhang, W.; Yang, R.; Cieplak, P.; Luo, R.; Lee, T. et al. A Point-Charge Force Field for Molecular Mechanics Simulations of Proteins Based on Condensed-Phase Quantum Mechanical Calculations. J. Comput. Chem. 2003, 24, 1999-2012.

(31) Phillips, J. C.; Braunand, R.; Wang, W.; Gumbart, J.; Tajkhorshid, E.; Villa, E.; Chipotand, C.; Skeel, R. D.; Kalé, L.; Schulten, K. Scalable Molecular Dynamics with NAMD. J. Comp. Chem. 2005, 26, 1781-1802.

(32) Abrahama, M. J.; Murtola, T.; Schulz, R.; Páll, S.; Smith, J. C.; Hess, B.; Lindahl, E. GROMACS: High Performance Molecular Simulations Through Multi-Level Parallelism from Laptops to Supercomputers. Software X 2015, 1-2, 19-25. 
(33) Svergun, D. I.; Koch, M. H. J. Small-Angle Scattering Studies of Biological Macromolecules in Solution. Rep. Prog. Phys. 2003, 66, 1735-1782.

(34) Marchi, M. A First Principle Particle Mesh Method for Solution SAXS of Large BioMolecular Systems. J. Chem. Phys. 2016, 145, 045101.

(35) Darden, T.; York, D.; Pedersen, L. Particle Mesh Ewald: An N $\log (\mathrm{N})$ Method for Ewald Sums in Large Systems. J. Chem. Phys. 1993, 98, 10089.

(36) Zhou, R.; Huang, X.; Margulis, C. J.; Berne, B. J. Hydrophobic Collapse in Multidomain Protein Folding. Science 2004, 305, 1605-1609.

(37) Chen, J.; Thirumalai, D. Interface Residues that Drive Allosteric Transitions Also Control the Assembly of L-Lactate Dehydrogenase. J. Phys. Chem. B 2018, 122, 11195-11205.

(38) Taguschi, H. The Simple and Unique Allosteric Machinery of Thermus Caldophilus Lactate Dehydrogenase, Structure-Function Relathionship in Bacterial Allosteric LDHs. Adv. Exp. Med. Biol. 2017, 925, 117-145.

(39) Monod, J.; Wyman, J.; Changeux, J.-P. On the Nature of Allosteric Transitions: A Plausible Model. J. Mol. Biol. 1965, 12, 88-118.

(40) Changeux, J.-P.; Edelstein, S. Conformational Selection or Induced Fit? 50 Years of Debate Resolved. F1000 Rep. Bio. 2011, 3, 19.

(41) Arai, K.; Ishimitsu, T.; Fushinobu, S.; Uchikoba, H.; Matsuzawa, H.; Taguchi, H. Active and Inactive State Structures of Unliganded Lactobacillus Casei Allosteric L-Lactate Dehydrogenase. Proteins 2009, 78, 681-694.

(42) Koide, S.; Yokoyama, S.; Matsuzawa, H.; Miyazawa, T.; Ohta, T. Conformational Equilibrium of an Enzyme Catalytic Site in the Allosteric Transition. Biochemistry 1992, 31, 5362-5368. 
(43) Colletier, J.; Aleksandrov, A.; Coquelle, N.; Mraihi, S.; Mendoza-Barberá, E.; Field, M.; Madern, D. Sampling the Conformational Energy Landscape of a Hyperthermophilic Protein by Engineering Key Substitutions. Mol. Biol. Evol. 2012, 29, 1683-1694. 

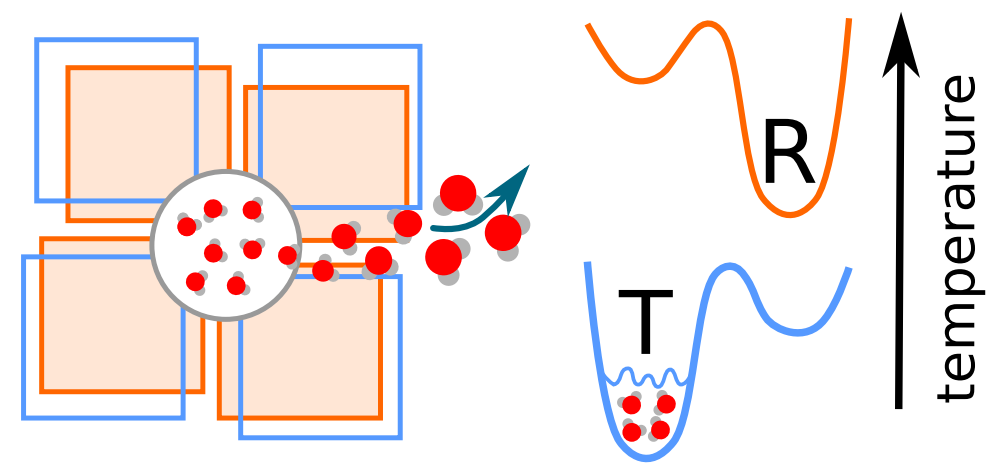

Figure 6: For Table of Contents Only 